\title{
THE TRANSFORMATION OF DOUBLE INTEGRALS
}

\author{
BY
}

R. G. HELSEL AND T. RADÓ

\section{Chapter I. Introduction}

1.1. Let there be given a continuous transformation $T$ of the form

$$
T: \quad x=x(u, v), \quad y=y(u, v),
$$

where $x(u, v), y(u, v)$ are defined and continuous on the unit square

$$
S_{0}: 0 \leqq u \leqq 1, \quad 0 \leqq v \leqq 1 .
$$

Designate the continuous image of the boundary of $S_{0}$ under $T$ by $C$. We shall be concerned with the validity of the topological transformation formula (apparently first studied by Schauder [1](1))

$$
\begin{aligned}
\iint_{S_{0}} F(x(u, v), y(u, v)) J(u, v ; T) d u d v \\
\quad=\int_{-\infty}^{+\infty} \int_{-\infty}^{+\infty} F(x, y) \mu\left(x, y ; S_{0}, T\right) d x d y,
\end{aligned}
$$

where $F(x, y)$ is any measurable function in the $x y$-plane; $J(u, v ; T)$ is the Jacobian, $x_{u}(u, v) y_{v}(u, v)-x_{v}(u, v) y_{u}(u, v)$; and $\mu\left(x, y ; S_{0}, T\right)$ is equal to the topological index of the point $(x, y)$ with respect to $C$ if $(x, y)$ is not on $C$; otherwise, $\mu\left(x, y ; S_{0}, T\right)=0$. The topological index of a point $(x, y)$ with respect to the continuous curve $C$ is defined as follows:

Definition. As $(u, v)$ describes the boundary of $S_{0}$ once in the counterclockwise sense, $(x(u, v), y(u, v))$ describes in the $x y$-plane the directed closed continuous curve $C$, and, if $(x, y)$ is a point not on $C$, the change of the continuously varying argument of the vector from $(x, y)$ to $(x(u, v), y(u, v))$ is of the form $2 k \pi$, where $k$ is an integer (positive, negative, or zero). The integer $k$ is called the topological index of the point $(x, y)$ with respect to $C$.

If $F(x, y) \equiv 1$, formula (1) becomes the area formula

$$
\iint_{S_{0}} J(u, v ; T) d u d v=\int_{-\infty}^{+\infty} \int_{-\infty}^{+\infty} \mu\left(x, y ; S_{0}, T\right) d x d y .
$$

If the transformation $T$ is biunique, $\left|\mu\left(x, y ; S_{0}, T\right)\right|$ assumes only two values, 0 or 1 , and formula (1) reduces to the ordinary transformation formula

Presented to the Society, April 24, 1943; received by the editors January 6, 1943.

(1) Numbers in brackets refer to bibliography at end of the paper. 


$$
\iint_{S_{0}} F(x(u, v), y(u, v))|J(u, v ; T)| d u d v=\iint_{T\left(S_{0}^{0}\right)} F(x, y) d x d y,
$$

where $T\left(S_{0}^{0}\right)$ is the image under $T$ of $S_{0}^{0}$, the set of interior points of $S_{0}$.

1.2. Radó and Reichelderfer have established formula (1), under the assumption that the integral on the left exists and the locus of $C$ is a set of planar measure zero, for a wide class of continuous transformations which they call $K_{3}$. (Many other results hold as well in class $K_{3}$. See Radó and Reichelderfer [1].) The purpose of the present paper is to show that this result implies all of the results on the transformation of double integrals in the literature of which we are aware (see McShane [1], Morrey [1], Rademacher [1], Radó [1, 2, 3], Schauder [1], Young [1, 2, 3]). It is not difficult to verify, by means of the two theorems of Radó and Reichelderfer quoted in 1.6 and 1.7, that the transformations considered by McShane [1], Morrey [1], Rademacher [1], Radó [1, 2, 3], and Schauder [1] belong to class $K_{3}$ and that the formulas established by these authors follow from formula (1). On the other hand, it is not apparent that Young's results are implied by those of Rado and Reichelderfer. We shall devote our attention, then, to the work of Young $[1,2,3]$, ultimately showing that all of his results can be accounted for in terms of the results of Radó and Reichelderfer. (It is interesting to note that our methods will place many of the transformations considered by Young in Morrey's class $L$. Morrey [1] has established formula (2) for transformations in class $L$.)

1.3. The two transformation formulas which Young develops are the same on the left as formulas (1) and (2); however, the right sides of Young's formulas are not Lebesgue integrals, but the limits of Lebesgue integrals. We shall now give a brief description of these limits. Let $\sigma$ be the generic notation for a subdivision of the boundary of $S_{0}$ by points $P_{1}, \cdots, P_{m}$, numbered consecutively in the positive sense around $S_{0}$. Let $\Pi_{\sigma}$ be the directed closed polygon "inscribed" in $C$ which is formed by straight segments connecting in order the images of $P_{1}, \cdots, P_{m}, P_{1}$ under $T$. Define $\mu_{\sigma}(x, y)$ to be equal to the topological index of the point $(x, y)$ with respect to $\Pi_{\sigma}$ if $(x, y)$ is not on $\Pi_{\sigma}$; otherwise, set $\mu_{\sigma}(x, y)=0$. Then if $F(x, y)$ is any measurable function in the $x y$-plane, Young defines the integral of $F(x, y)$ over the area of the polygon $\Pi_{\sigma}$ as follows:

$$
\text { (Y) } \iint_{\mathbf{I}_{\sigma}} F(\dot{x}, y) d x d y=\int_{-\infty}^{+\infty} \int_{-\infty}^{+\infty} F(x, y) \mu_{\sigma}(x, y) d x d y,
$$

providing the Lebesgue integral on the right exists. Young then defines the integral of $F(x, y)$ over the area of the curve $C$ in the following way:

$$
\text { (Y) } \iint_{C} F(x, y) d x d y=\lim _{\|\boldsymbol{\sigma}\| \rightarrow 0}(Y) \iint_{\Pi_{\sigma}} F(x, y) d x d y,
$$


providing the limit on the right exists and is independent of the manner in which $\|\sigma\| \rightarrow 0$. $(\|\sigma\|$ is the norm of the subdivision $\sigma$.

1.4. For certain classes of continuous transformations

$$
T: x=x(u, v), \quad y=y(u, v), \quad(u, v) \in S_{0},
$$

Young has established the area formula

$$
\iint_{S_{0}} J(u, v ; T) d u d v=(Y) \iint_{C} d x d y,
$$

where $C$ is the image of the boundary of $S_{0}$ under $T$ and $(Y) \iint_{C} d x d y$ is assumed to exist. In a few instances, he also asserts that the general transformation formula

$$
\iint_{S_{0}} F(x(u, v), y(u, v)) J(u, v ; T) d u d v=(Y) \iint_{C} F(x, y) d x d y
$$

holds for any measurable function $F(x, y)$ in the $x y$-plane as soon as the integral on the right exists. Throughout his work, Young assumes that the coordinate functions $x(u, v)$ and $y(u, v)$ of the transformation $T$ are absolutely continuous on every horizontal and on every vertical in $S_{0}$; hence, the continuous curve $C$ is rectifiable and its locus is a set of planar measure zero.

1.5. A comparison of formulas (1) and (4) leads to this engaging question: When does

$$
\int_{-\infty}^{+\infty} \int_{-\infty}^{+\infty} F(x, y) \mu_{\sigma}(x, y) d x d y \underset{\|\sigma\| \rightarrow 0}{\longrightarrow} \int_{-\infty}^{+\infty} \int_{-\infty}^{+\infty} F(x, y) \mu\left(x, y ; S_{0}, T\right) d x d y ?
$$

Young neither raises nor answers this question. Instead, he always assumes the existence of the limit on the left and defines it to be the new integral, $(Y) \iint_{C} F(x, y) d x d y$. It is clear that an improvement is achieved if in formula (4) we can replace $(Y) \iint_{C} F(x, y) d x d y$ by $\int_{-\infty}^{+\infty} \int_{-\infty}^{+\infty} F(x, y) \mu\left(x, y ; S_{0}, T\right) d x d y$. (Schauder [1] has already enunciated the desideratum of replacing $(Y) \iint_{C} F(x, y) d x d y$ by something more tangible.) In fact, in every case considered by Young but one, we shall accomplish this replacement by showing that the transformation belongs to class $K_{3}$ and, hence, formula (1) holds as soon as the integral on the left exists (cf. 1.2, 1.4). In the one exceptional case just mentioned, it would seem that Young did not establish anything (cf. 3.10). (The preceding statements should not be construed to mean that we have succeeded in identifying $(Y) \iint_{C} F(x, y) d x d y$ and $\int_{-\infty}^{+\infty} \int_{-\infty}^{+\infty} F(x, y) \mu\left(x, y ; S_{0}, T\right) d x d y$ in all the cases of Young. Actually, it appears to us that a general study of the relation of these integrals would lead to questions of great difficulty and interest.)

1.6. To place Young's transformations in class $K_{3}$, we shall utilize the two following theorems of Radó and Reichelderfer [1]. 
Theorem. If the transformation $T$ is continuous on the domain $\mathcal{D}$ of the $u v$-plane; the partial derivatives $x_{u}(u, v), x_{v}(u, v), y_{u}(u, v), y_{v}(u, v)$ exist and are continuous on $\mathcal{D}$; and $J(u, v ; T)$ is summable on $\mathcal{D}$, then $T \in K_{3}(\mathcal{D})$.

1.7. Closure theorem. Let there be given bounded domains $\mathcal{D}$ and $\mathcal{D}_{n}$ in the uv-plane and continuous transformations

$$
\begin{array}{lllrl}
T: & x=x(u, v), & y=y(u, v), & (u, v) \in \mathcal{D}, \\
T_{n}: & x=x_{n}(u, v), & y=y_{n}(u, v), & (u, v) \in \mathcal{D}_{n},
\end{array}
$$

with the following properties: (i) $\mathcal{D}_{n} \subset \mathcal{D}$ and, for every closed set $F \subset \mathcal{D}$, there exists an $n_{0}=n_{0}(F)$ such that $F \subset \mathcal{D}_{n}$ for all $n>n_{0}$; (ii) $J(u, v ; T$ ) exists a.e. (almost everywhere) and is summable on $\mathcal{D}$; (iii) $T_{n} \in K_{3}\left(\mathcal{D}_{n}\right)$ for $n=1,2, \cdots$;

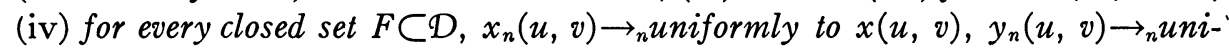
formly to $y(u, v)$, on $F$ and

$$
\lim _{n} \iint_{F}\left|J\left(u, v ; T_{n}\right)-J(u, v ; T)\right| d u d v=0 .
$$

Then $T \in K_{3}(\mathcal{D})$.

(The Closure Theorem is still valid if the condition

$$
\lim _{n} \iint_{F}\left|J\left(u, v ; T_{n}\right)-J(u, v ; T)\right| d u d v=0
$$

is replaced by the weaker condition

$$
\left.\lim _{n} \iint_{F}\left|J\left(u, v ; T_{n}\right)\right| d u d v=\iint_{F}|J(u, v ; T)| d u d \vartheta .\right)
$$

1.8. The method employed in Chapter III to place each transformation considered by Young in class $K_{3}$ is this: We approximate to the given transformation by a sequence of transformations which belong to class $K_{3}$ (to begin, we use transformations which belong to class $K_{3}$ because of 1.6) and satisfy the conditions of the Closure Theorem, thus placing the given transformation in class $K_{3}$. The feature of this paper is the utilization of integral means to obtain the approximating transformations.

The second chapter of this paper is a systematic presentation of general theorems in analysis and properties of integral means which we apply in Chapter III to prove our results. Since integral means give rise to nondenumerable sequences of approximating functions, many of the theorems in Chapter II are concerned with such sequences; however, we work only with denumerable sequences in Chapter III. The intrinsic interest of our results on non-denumerable sequences of integral means justifies their inclusion.

1.9. The cases which we treat in Chapter III are numerous and contain many assumptions so we shall not list them here, but we shall summarize 
the essential differences between our results and those of Young.

1. By placing Young's transformations in class $K_{3}$, we do more than establish formula (1) of 1.1 for them, since many other results hold as well for transformations in class $K_{3}$ (cf. 1.2).

2. In most of his cases, Young did not establish formula (4) of 1.4, but only the area formula (3). On the other hand, we shall establish the general topological transformation formula (1) for all of Young's transformations. Young established formula (3) for the transformations considered in 3.6, 3.7, $3.9,3.12$, and 3.14; formula (4) for the transformations of 3.8 and 3.14.1. The transformation of 3.14.1 is the most general case for which Young claims to have established formula (4).

3. Throughout Chapter III we assume there is given a continuous transformation $T: x=x(u, v), y=y(u, v),(u, v) \in S_{0}$, which satisfies the standard hypothesis $H_{0}: x(u, v)$ and $y(u, v)$ are absolutely continuous in the Tonelli sense (cf. 2.17) on $S_{0}$ and the image of the boundary of $S_{0}$ under $T$ is a set of planar measure zero. The additional restrictions on $T$ which we use in Chapter III do not differ from restrictions used by Young; however, Young always makes the standard assumption that $x(u, v)$ and $y(u, v)$ are of bounded variation in the Tonelli sense (cf. 2.17) on $S_{0}$ and are absolutely continuous on every horizontal and on every vertical in $S_{0}$. Clearly our hypothesis $H_{0}$ is less restrictive than Young's standard assumption (cf. 1.4).

It must be remembered that in all cases our theorems are not identical with those of Young (cf. 1.3, 1.5). The references to Young's work which appear in 3.6, 3.7, 3.8, 3.9, 3.12,3.14, and 3.14.1 direct the reader to the theorems of Young which correspond to our theorems of those sections.

1.10. Young's theorems on the transformation of double integrals are not final in character; hence, we have attempted to find a general theorem which would at once account for all of his results. The following proposition, which we have not been able to establish or deny, seems a likely generalization (cf. 3.15):

If $T$ satisfies $H_{0}(c f .1 .9)$ and $J(u, v ; T)$ is summable on $S_{0}$, then $T \in K_{3}\left(S_{0}^{0}\right)$ and formula (1) holds if the integral on the left exists.

\section{Chapter II. Auxiliary theorems and integral means}

2.1. Most of the results listed in this chapter are known; however, a few of them are new-for example, see 2.12, 2.21, 2.21.1. Proofs are included whenever convenient references are not available.

2.2. The following concept will be used extensively in the present paper:

Definition. A family $\mathcal{F}$ of measurable functions defined on $S_{0}$ is said to have the Vitali property-briefly, property (U)—on a measurable subset $E$ of $S_{0}^{0}$ if the following condition is satisfied. To every $\epsilon>0$ there corresponds $a \delta=\delta(\epsilon)$ $>0$ such that 


$$
\left|\iint_{e} f(u, v) d u d v\right|<\epsilon
$$

for every function $f(u, v)$ of $\mathcal{F}$ and every measurable set $e \subset E$ with $|e|<\delta$. (If e is any set, $|e|$ denotes the exterior measure of $e$.

2.2.1. CoRollary. If a family $\mathcal{F}$ of functions $f(u, v)$ has property $(U)$ on a measurable subset $E$ of $S_{0}$, then the family $\mathcal{F}^{*}$ of functions $|f(u, v)|$ also has, and conversely.

2.2.2. COROLlaRY. If $\mathcal{Z}$ is a family of functions possessing property (U) on a measurable subset $E$ of $S_{0}$ and $G$ is a family of measurable functions which are uniformly bounded on $E$, then the family of functions $f(u, v) \cdot g(u, v), f(u, v) \in \mathcal{F}$ and $g(u, v) \in G$, has property $(U)$ on $E$.

2.2.3. CoRollary. If a family $\mathcal{F}$ of functions has property $(\mathcal{U})$ on a measurable subset $E$ of $S_{0}$, then $\iint_{E} f(u, v) d u d v$ exists for every function $f(u, v)$ of $\mathcal{F}$ and these integrals are uniformly bounded.

2.3. ThEOREM. If on a measurable subset $E$ of $S_{0}, f_{n}(u, v) \geqq 0$ and $f_{n}(u, v)$ $\rightarrow_{n} f(u, v)$ a.e., a necessary and sufficient condition for $f(u, v)$ to be summable on E and

$$
\iint_{E} f_{n}(u, v) d u d v \underset{n}{\rightarrow} \iint_{E} f(u, v) d u d v
$$

is that $\left\{f_{n}(u, v)\right\}$ have property $(\mho)$ on $E$.

Proof. See de la Vallée Poussin [1, p. 477]. This theorem may be extended to a non-denumerable sequence of continuous functions $f_{\alpha}(u, v), 0<\alpha<1$, such that $f_{\alpha}(u, v) \rightarrow_{\alpha \rightarrow 0} f(u, v)$ a.e. on $E$.

2.3.1. Corollary. If on a measurable subset $E$ of $S_{0}, f_{n}(u, v) \rightarrow_{n} f(u, v)$ a.e., a necessary and sufficient condition for $f(u, v)$ to be summable on $E$ and

$$
\iint_{E}\left|f_{n}(u, v)-f(u, v)\right| d u d v \underset{n}{\rightarrow} 0
$$

is that $\left\{f_{n}(u, v)\right\}$ have property $(\mathcal{U})$ on $E$.

2.4. Many of the properties of integral means which we shall utilize depend upon the next two theorems concerning partial differentiation of an indefinite integral.

THEOREM. If $f(u, v)$ is summable on $S_{0}$ and

$$
F(u, v)=\int_{0}^{u} \int_{0}^{v} f(\xi, \eta) d \xi d \eta, \quad(u, v) \in S_{0}
$$


then, for every $v, F_{u}(u, v)=\int_{0}^{v} f(u, \eta) d \eta$, provided $u$ does not belong to a set of measure zero which is independent of $v$.

Proof. See Fubini. [1]. (Similarly, for every $u, F_{v}(u, v)=\int_{0}^{u} f(\xi, v) d \xi$, provided $v$ does not belong to a set of measure zero which is independent of $u$. A majority of the theorems of this chapter are stated in an unsymmetric form as regards $u$ and $v$. In each such instance, a second theorem may be obtained by interchanging the rôles of $u$ and $v$.)

2.5. ThEOREM. If $f(u, v)$ is summable on $S_{0}$ and

$$
g(u, v)=\int_{0}^{u} f(\xi, v) d \xi, \quad(u, v) \in S_{0},
$$

(for a.e. $v, g(u, v)$ exists for every $u$ ) then $g_{u}(u, v)=f(u, v)$ a.e. on $S_{0}$.

Proof. See Helsel and Young [1].

2.6. We shall have occasion to employ the following types of integral means.

Definition. If $f(u, v)$ is summable on $S_{0}$ and $0<h<1 / 2$ is fixed, then

$$
f_{h}^{h}(u, v)=\frac{1}{4 h^{2}} \int_{-h}^{h} \int_{-h}^{h} f(u+\alpha, v+\beta) d \alpha d \beta=\frac{1}{4 h^{2}} \int_{u-h}^{u+h} \int_{v-h}^{v+h} f(\xi, \eta) d \xi d \eta,
$$

defined on the square $S_{h}: h \leqq u \leqq 1-h, h \leqq v \leqq 1-h$, is called the $h$-h-integral mean of $f(u, v)$.

Definition. If $f(u, v)$ is summable on $S_{0}$ and $0<h<1 / 2$ is fixed, then

$$
f_{h}(u, v)=\frac{1}{2 h} \int_{-h}^{h} f(u+\alpha, v) d \alpha=\frac{1}{2 h} \int_{u-h}^{u+h} f(\xi, v) d \xi
$$

defined for a.e. $v$ on the rectangle $R_{h 0}: h \leqq u \leqq 1-h, 0 \leqq v \leqq 1$, is called the $h$-integral mean of $f(u, v)$.

2.7. TheOREM. If $f(u, v)$ is continuous on $S_{0}$ and $R$ (a closed oriented rectangle) comprised in $S_{0}^{0}$ is fixed, then, as $h \rightarrow 0, f_{h}^{h}(u, v) \rightarrow$ uniformly to $f(u, v)$ on $R$.

Proof. Well known properties of integral means such as this are established in Bray [1], Morrey [1], and Radó [2]. When theorems on integral means are stated without proofs or references, the reader should consult the above papers.

2.8. ThEOREM. If $f(u, v)$ is continuous on $S_{0}$, then on $S_{h}$

$$
\frac{\partial f_{h}^{h}(u, v)}{\partial u}=\frac{1}{4 h^{2}} \int_{v-h}^{v+h}\{f(u+h, \eta)-f(u-h, \eta)\} d \eta .
$$


2.9. TheOREM. If $f(u, v)$ is summable on $S_{0}$, then $f_{h}^{h}(u, v) \rightarrow_{h \rightarrow 0} f(u, v)$ a.e. on $S_{0}$.

Proof. The result is immediate from Lebesgue's theorem on absolutely continuous set functions. See de la Vallée Poussin [1].

2.10. Theorem. If $f(u, v)$ is summable on $S_{0}$ and $R \subset S_{0}^{0}$ is fixed, then $\left\{f_{h}^{h}(u, v)\right\}$ has property $(U)$ on $R$.

Proof. Let $e$ be any measurable set comprised in $R$. Then for $h$ small enough that $R \subset S_{h}$, we have

$$
\begin{aligned}
\left|\iint_{e} f_{h}^{h}(u, v) d u d v\right| & \leqq \frac{1}{4 h^{2}} \int_{-h}^{h} \int_{-h}^{h}\left\{\iint_{e}|f(u+\alpha, v+\beta)| d u d v\right\} d \alpha d \beta \\
& \leqq \frac{1}{4 h^{2}} \int_{-h}^{h} \int_{-h}^{h}\left\{\iint_{e_{\alpha}^{\beta}}|f(u, v)| d u d v\right\} d \alpha d \beta \\
& \leqq \frac{1}{4 h^{2}} \int_{-h}^{h} \int_{-h}^{h} \Omega\left(|f|, S_{0},|e|\right) d \alpha d \beta=\Omega\left(|f|, S_{0},|e|\right),
\end{aligned}
$$

where

$$
\begin{gathered}
e_{\alpha}^{\beta}=\underset{(u, v)}{E}[(u-\alpha, v-\beta) \in e], \\
\Omega\left(|f|, S_{0},|e|\right)=\max _{E \text { meas } \subset S_{0},|E| \leqq|e|} \iint_{E}|f(u, v)| d u d v .
\end{gathered}
$$

Because of the absolute continuity of $\iint_{E}|f(u, v)| d u d v$ as a set function on $S_{0}$, $\Omega\left(|f|, S_{0},|e|\right) \rightarrow_{|e| \rightarrow 0} 0$; hence, $\left\{f_{h}^{h}(u, v)\right\}$ has property $(U)$ on $R$.

2.11. THEOREM. If $f(u, v)$ and $g(u, v)$ belong to associated Lebesgue classes $L^{p}$ and $L^{q}$ respectively, where $p>0, q>0,1 / p+1 / q=1$, and if $R \subset S_{0}^{0}$ is fixed, then $\left\{f_{h}^{h}(u, v) \cdot g_{h}^{h}(u, v)\right\}$ has property $(U)$ on $R$.

Proof. The result follows from Hölder's inequality and reasoning similar to that employed in 2.10 .

2.12. TheOREM. If $f(u, v)$ is summable on $S_{0}$ and the closed interval $(a, b)$ is strictly interior to $(0,1)$, then

$$
\int_{a}^{b}\left|f_{h}^{h}(u, v)\right| d u \underset{h \rightarrow 0}{\longrightarrow} \int_{a}^{b}|f(u, v)| d u,
$$

provided $v$ does not belong to a set of measure zero which is independent of $a$ and $b$.

Proof. By 2.9, $f_{h}^{h}(u, v) \rightarrow_{h \rightarrow 0} f(u, v)$ a.e. on $S_{0}$; hence from the Theorem of Fubini, $f_{h}^{h}(u, v) \rightarrow_{h \rightarrow 0} f(u, v)$ a.e. on a.e. line $v=$ constant in $S_{0}$. Let $E_{v}^{1}$ be the exceptional set of $v$ values. Also, by the Theorem of Fubini, $\int_{0}^{1}|f(u, v)| d u$ 
exists for $v$ not belonging to an exceptional set $E_{v}^{2}$ of measure zero.

Choose two rational numbers $\alpha$ and $\beta$ such that $0<\alpha<\beta<1$. Then define

$$
g(v)=\int_{\alpha-\delta}^{\beta+\delta}|f(u, v)| d u, \quad v \operatorname{not} \in E_{v}^{2},
$$

where $\delta>0$ is rational and $0 \leqq \alpha-\delta<\beta+\delta \leqq 1$. Since $g(v)$ is summable on $0 \leqq v \leqq 1$, it follows from Lebesgue's theorem on absolutely continuous set functions that

$$
\frac{1}{2 h} \int_{-h}^{h} g(v+\eta) d \eta \underset{h \rightarrow 0}{\longrightarrow} g(v),
$$

for $v$ not belonging to an exceptional set $E_{v}^{3}(\alpha, \beta, \delta)$ of measure zero. Put

$$
E_{v}=E_{v}^{1}+E_{v}^{2}+\sum_{\alpha, \beta, \delta \text { rat. }} E_{v}^{3}(\alpha, \beta, \delta)
$$

Then $\left|E_{v}\right|=0$.

For $v$ not $\in E_{v}$ and for a fixed rational $\delta>h$,

$$
\begin{aligned}
\int_{\alpha}^{\beta}\left|f_{h}^{h}(u, v)\right| d u & \leqq \frac{1}{4 h^{2}} \int_{-h}^{h} \int_{-h}^{h}\left\{\int_{\alpha}^{\beta}|f(u+\xi, v+\eta)| d u\right\} d \xi d \eta \\
& \leqq \frac{1}{4 h^{2}} \int_{-h}^{h} \int_{-h}^{h}\left\{\int_{\alpha-\delta}^{\beta+\delta}|f(u, v+\eta)| d u\right\} d \xi d \eta \\
& \leqq \frac{1}{2 h} \int_{-h}^{h} g(v+\eta) d \eta .
\end{aligned}
$$

Therefore

$$
\underset{h \rightarrow 0}{\limsup } \int_{\alpha}^{\beta}\left|f_{h}^{h}(u, v)\right| d u \leqq g(v)=\int_{\alpha-\delta}^{\beta+\delta}|f(u, v)| d u, v \text { not } \in E_{v} .
$$

As $\delta \rightarrow 0$ through positive rational values, we obtain

$$
\limsup _{h \rightarrow 0} \int_{\alpha}^{\beta}\left|f_{h}^{h}(u, v)\right| d u \leqq \int_{\alpha}^{\beta}|f(u, v)| d u, \quad v \operatorname{not} \in E_{v} .
$$

This inequality holds for any two real numbers $0<a<b<1$, since for $v$ not $\in E_{v}$

$$
\begin{aligned}
\limsup _{h \rightarrow 0} \int_{a}^{b}\left|f_{h}^{h}(u, v)\right| d u & \leqq \limsup _{n \rightarrow 0} \int_{\alpha_{n}}^{\beta_{n}}\left|f_{h}^{h}(u, v)\right| d u \\
& \leqq \int_{\alpha_{n}}^{\beta_{n}}|f(u, v)| d u \underset{n}{\rightarrow} \int_{a}^{b}|f(u, v)| d u,
\end{aligned}
$$

where $\left\{\alpha_{n}\right\}$ and $\left\{\beta_{n}\right\}$ are sequences of rational numbers converging to $a$ and $b$ respectively and satisfying $0<\alpha_{n}<a<b<\beta_{n}<1$. 
By the Lemma of Fatou, for any two real numbers $0<a<b<1$

$$
\begin{aligned}
\int_{a}^{b}|f(u, v)| d u & =\int_{a}^{b} \liminf _{h \rightarrow 0}\left|f_{h}^{h}(u, v)\right| d u \\
& \leqq \liminf _{h \rightarrow 0} \int_{a}^{b}\left|f_{h}^{h}(u, v)\right| d u, \quad v \text { not } \in E_{v}^{1}+E_{v}^{2}
\end{aligned}
$$

The result follows from (1) and (2).

2.13. LEMmA. On $a \leqq u \leqq b$, let there be given a family of functions $f_{\alpha}(u)$, $0<\alpha<1$, which converge a.e. to $f(u)$ as $\alpha \rightarrow 0$. If, for $0<\alpha<1$ and $a \leqq u \leqq b$, $\left|f_{\alpha}(u)\right| \leqq g(u)$, where $g(u)$ is summable on $a \leqq u \leqq b$, then $\int_{a}^{b} f_{\alpha}(u) d u \rightarrow \rightarrow_{\alpha \rightarrow 0} \int_{a}^{b} f(u) d u$.

Proof. Deny the truth of the assertion. Then there exists a denumerable subsequence of functions $\left\{f_{\alpha_{n}}(u)\right\}$ such that $f_{\alpha_{n}}(u) \rightarrow_{n} f(u)$ a.e. on $a \leqq u \leqq b$ and $\int_{a}^{b} f_{\alpha_{n}}(u) d u$ does not converge to $\int_{a}^{b} f(u) d u$. This contradicts Lebesgue's theorem on termwise integration.

2.14. TheOREM. If $f(u, v) \geqq 0$ is summable on $S_{0}, \lambda(u) \geqq 0$ is summable on $0 \leqq u \leqq 1$, and $V(u)=\int_{0}^{1} f(u, v) d v$ is $a$ bounded function of $u$ (on the set where it exists), then for a fixed rectangle $R: 0<a \leqq u \leqq b<1,0<c \leqq v \leqq d<1$,

$$
\iint_{R} f_{h}^{h}(u, v) \lambda(u) d u d v \underset{h \rightarrow 0}{\longrightarrow} \iint_{R} f(u, v) \lambda(u) d u d v .
$$

Proof. Choose $h$ small enough that $R \subset S_{h}$. By 2.12 , for a.e. $u, a \leqq u \leqq b$,

$$
\lambda(u) \int_{c}^{d} f_{h}^{h}(u, v) d v \underset{h \rightarrow 0}{\longrightarrow} \lambda(u) \int_{c}^{d} f(u, v) d v .
$$

Also

$$
\begin{aligned}
\lambda(u) \int_{c}^{d} f_{h}^{h}(u, v) d v & =\frac{\lambda(u)}{4 h^{2}} \int_{-h}^{h} \int_{-h}^{h}\left\{\int_{c}^{d} f(u+\alpha, v+\beta) d v\right\} d \alpha d \beta \\
& \leqq \frac{\lambda(u)}{4 h^{2}} \int_{-h}^{h} \int_{-h}^{h} V(u+\alpha) d \alpha d \beta \leqq M \cdot \lambda(u),
\end{aligned}
$$

where $M$ is a bound for $V(u)$. By the lemma of 2.13

$$
\int_{a}^{b}\left\{\lambda(u) \int_{c}^{d} f_{h}^{h}(u, v) d v\right\} d u \underset{h \rightarrow 0}{\longrightarrow} \int_{a}^{b}\left\{\lambda(u) \int_{c}^{d} f(u, v) d v\right\} d u .
$$

Hence, by the Theorem of Tonelli,

$$
\iint_{R} f_{h}^{h}(u, v) \lambda(u) d u d v \underset{h \rightarrow 0}{\longrightarrow} \iint_{R} f(u, v) \lambda(u) d u d v .
$$


2.15. THEOREM. If $f(u, v)$ is continuous on $S_{0}$, then on $R_{h 0}(c f .26)$

$$
\frac{\partial f_{h}(u, v)}{\partial u}=\frac{1}{2 h}\{f(u+h, v)-f(u-h, v)\} .
$$

2.16. TheOREM. If $f(u, v)$ is summable on $S_{0}$, then $f_{h}(u, v) \rightarrow_{h \rightarrow 0} f(u, v)$ a.e. on $S_{0}$.

Proof. The result is immediate from 2.5.

2.17. For the purpose of Chapter III, we shall need certain theorems pertaining to functions which are absolutely continuous in the sense of Tonellibriefly, A.C.T.-and to their integral means.

Definition. The function $f(u, v)$ is A.C.T. on $S_{0}$ if (i) $f(u, v)$ is continuous; (ii) $f(u, v)$ is of bounded variation in the sense of Tonelli; (iii) for a.e. $v=\eta$, $0 \leqq \eta \leqq 1$, the function $f(u, \eta)$ is absolutely continuous in $u$, and, for a.e. $u=\xi$, $0 \leqq \xi \leqq 1$, the function $f(\xi, v)$ is absolutely continuous in $v$. Denote by $V(\eta ; f)$ the total variation of $f(u, \eta)$ as a function of $u$ on $0 \leqq u \leqq 1$; denote by $V(\xi ; f)$ the total variation of $f(\xi, v)$ as a function of $v$ on $0 \leqq v \leqq 1$. Then $f(u, v)$ is of bounded variation in the sense of Tonelli if $V(\eta ; f)$ and $V(\xi ; f)$ are summable functions of $\eta$ and $\xi$ respectively on the interval $(0,1)$.

2.18. If $f(u, v)$ is A.C.T. on $S_{0}$, the partial derivatives $f_{u}(u, v)$ and $f_{v}(u, v)$ exist a.e. and are summable on $S_{0}$; moreover, for a.e. $v, V(v ; f)=\int_{0}^{1}\left|f_{u}(u, v)\right| \partial u$ and, for a.e. $u, V(u ; f)=\int_{0}^{1}\left|f_{v}(u, v)\right| d v$ (see Morrey [1]).

2.19. ThEOREM. If $f(u, v)$ is A.C.T. on $S_{0}$, then $\partial f_{h}^{h}(u, v) / \partial u=(\partial f(u, v) / \partial u)_{h}^{h}$ on $S_{h}$.

2.19.1. CoRollaRy. If $f(u, v)$ is A.C.T.on $S_{0}$, then $\partial f_{h}^{h}(u, v) / \partial u \rightarrow_{h \rightarrow 0} \partial f(u, v) / \partial u$ a.e. on $S_{0}$.

Proof. The result is immediate from 2.19 and 2.9.

2.20. THEOREM. If $f(u, v)$ is A.C.T. on $S_{0}$, then $\partial f_{h}(u, v) / \partial u=(\partial f(u, v) / \partial u)_{h}$ on $R_{h 0}$ providing $v$ does not belong to a set of measure zero which is independent of $h$.

Proof. By 2.15, $\partial f_{h}(u, v) / \partial u=(1 / 2 h)\{f(u+h, v)-f(u-h, v)\}$ on $R_{h 0}$ Since $f(u, v)$ is A.C.T., it is absolutely continuous in $u$ for a.e. $v, 0 \leqq v \leqq 1$. Fix a value of $v$ for which $f(u, v)$ is absolutely continuous in $u$. Then

$$
\begin{aligned}
\left(\frac{\partial f(u, v)}{\partial u}\right)_{h} & =\frac{1}{2 h} \int_{u-h}^{u+h} \frac{\partial f(\xi, v)}{\partial \xi} d \xi \\
& =\frac{1}{2 h}\{f(u+h, v)-f(u-h, v)\}=\frac{\partial f_{h}(u, v)}{\partial u} .
\end{aligned}
$$


2.20.1. Corolla RY. If $f(u, v)$ is A.C.T.on $S_{0}$, then $\partial f_{h}(u, v) / \partial u \rightarrow_{h \rightarrow 0} \partial f(u, v) / \partial u$ a.e. on $S_{0}$.

Proof. The result is immediate from 2.20 and 2.16 .

2.21. Theorem. If $f(u, v)$ is A.C.T. on $S_{0}$, then $\partial f_{h}(u, v) / \partial v=(\partial f(u, v) / \partial v)_{h}$ on $R_{h 0}$ providing $v$ does not belong to a set of measure zero which is independent of $h$.

Proof. For $0 \leqq v \leqq 1$,

$$
\begin{aligned}
\int_{0}^{v}\left(\frac{\partial f(u, \eta)}{\partial \eta}\right)_{h} d \eta & =\frac{1}{2 h} \int_{-h}^{h}\left\{\int_{0}^{v} \frac{\partial f(u+\alpha, \eta)}{\partial \eta} d \eta\right\} d \alpha \\
& =\frac{1}{2 h} \int_{-h}^{h}\{f(u+\alpha, v)-f(u+\alpha, 0)\} d \alpha \\
& =f_{h}(u, v)-f_{h}(u, 0) .
\end{aligned}
$$

Therefore, on $\dot{R}_{h 0}$,

$$
f_{h}(u, v)=f_{h}(u, 0)+\frac{1}{2 h} \int_{0}^{v} \int_{u-h}^{u+h} \frac{\partial f(\xi, \eta)}{\partial \eta} d \xi d \eta .
$$

By 2.4,

$$
\frac{\partial f_{h}(u, v)}{\partial v}=\frac{1}{2 h} \int_{u-h}^{u+h} \frac{\partial f(\xi, v)}{\partial v} d \xi=\left(\frac{\partial f(u, v)}{\partial v}\right)_{h}
$$

on $R_{h 0}$ providing $v$ does not belong to a set of measure zero which is independent of $h$.

2.21.1. Corollary. If $f(u, v)$ is A.C.T. on $S_{0}$, then $\partial f_{h}(u, v) / \partial v \rightarrow_{h \rightarrow 0} \partial f(u, v) / \partial v$ a.e. on $S_{0}$.

2.22. Theorem. If $f(u, v)$ is A.C.T. on $S_{0}$, then $f_{h}(u, v)$ is A.C.T. on $R_{h 0}$.

Proof. Because of the absolute continuity of the indefinite Lebesgue integral, $f_{h}(u, v)$ is absolutely continuous in $u$ for every $v, 0 \leqq v \leqq 1$; hence,

$$
\begin{aligned}
V\left(v ; f_{h}\right) & =\int_{h}^{1-h}\left|\frac{\partial f_{h}(u, v)}{\partial u}\right| d u \\
& =\frac{1}{2 h} \int_{h}^{1-h}|f(u+h, v)-f(u-h, v)| d u \leqq \frac{M}{h}
\end{aligned}
$$

where $M$ is a bound for $|f(u, v)|$ on $S_{0}$. Thus $V\left(v ; f_{h}\right)$ is summable on $0 \leqq v \leqq 1$.

From the proof of 2.21 , on $R_{h 0}$,

$$
f_{h}(u, v)=f_{h}(u, 0)+\frac{1}{2 h} \int_{0}^{v}\left\{\int_{u-h}^{u+h} \frac{\partial f(\xi, \eta)}{\partial \eta} d \xi\right\} d \eta .
$$


Therefore, $f_{h}(u, v)$ is absolutely continuous in $v$ for every $u, h \leqq u \leqq 1-h$, and

$$
\begin{aligned}
V\left(u ; f_{h}\right) & =\int_{0}^{1}\left|\frac{\partial f_{h}(u, v)}{\partial v}\right| d v=\int_{0}^{1}\left|\left(\frac{\partial f(u, v)}{\partial v}\right)_{h}\right| d v \\
& \leqq \frac{1}{2 h} \int_{0}^{1}\left\{\int_{u-h}^{u+h}\left|f_{v}(\xi, v)\right| d \xi\right\} d v \\
& \leqq \frac{1}{2 h} \iint_{S_{0}}\left|f_{v}(u, v)\right| d u d v .
\end{aligned}
$$

Thus $V\left(u ; f_{h}\right)$ is summable on $h \leqq u \leqq 1-h$ and the proof is complete.

Chapter III. Transformation formulas for DOUble integrals

3.1. In this final chapter we shall take each transformation for which W. H. Young established either formula (3) or (4) of 1.4 and, by placing it in class $K_{3}$, show that formula (1) of 1.1 holds if the integral on the left exists (cf. 1.2). Throughout the chapter we make the assumption that there is given a transformation

$$
T: \quad x=x(u, v), \quad y=y(u, v), \quad(u, v) \in S_{0},
$$

which satisfies hypothesis $H_{0}$ of 1.9. Additional restrictions on the transformation $T$ will be stated in the sequel. In the last three theorems of this chapter (cf. 3.9, 3.12,3.14), the conditions imposed on the coordinate functions $x(u, v)$ and $y(u, v)$ are not symmetric. A second theorem may be obtained in each of these cases by interchanging the rôles of $x(u, v)$ and $y(u, v)$.

3.2. We shall now show that certain transformations obtained from $T$ by approximating to the functions $x(u, v), y(u, v)$ with $h$-h-integral means belong to class $K_{3}$. First we introduce the following notations:

${ }_{n}^{n} f(u, v)=f_{1 / n}^{1 / n}(u, v), \quad{ }_{n} f(u, v)=f_{1 / n}(u, v), \quad{ }_{n} S=S_{1 / n}, \quad n=3,4, \cdots$, where $f(u, v)$ is any summable function defined on $S_{0}$.

3.3. LEMмA. If $T$ satisfies $H_{0}$, the transformation

$$
{ }_{n}^{n} T_{m}^{m}: \quad x={ }_{n}^{n} x(u, v), \quad y={ }_{m}^{m} y(u, v), \quad n, m>2,(u, v) \in{ }_{n} S \cdot{ }_{m} S,
$$

belongs to class $K_{3}$ on ${ }_{n} S^{0} \cdot{ }_{m} S^{0}$.

Proof. By 2.8, $\partial_{n}^{n} x(u, v) / \partial u, \partial_{n}^{n} x(u, v) / \partial v, \partial_{m}^{m} y(u, v) / \partial u, \partial_{m}^{m} y(u, v) / \partial v$ are continuous on ${ }_{n} S \cdot{ }_{m} S$; hence, ${ }^{n} J^{m}=J\left(u, v ;{ }_{n}^{n} T_{m}^{m}\right)$ is summable there. By 1.6, ${ }_{n}^{n} T_{m}^{m} \in K_{3}\left({ }_{n} S^{0} \cdot{ }_{m} S^{0}\right)$.

\subsubsection{Corollary. If $T$ satisfies $H_{0}$, the transformation}

$$
{ }_{n}^{n} T_{n}^{n}: \quad x={ }_{n}^{n} x(u, v), \quad y={ }_{n}^{n} y(u, v), \quad n>2,(u, v) \in{ }_{n} S,
$$


belongs to class $K_{3}$ on ${ }_{n} S^{0}$.

3.3.2. CoROllaRy. If $T$ satisfies $H_{0}$, the transformation ${ }_{n}^{n} T_{m}^{m}(1 / n+1 / m<1 / 2)$ belongs to class $K_{3}$ on $S_{1 / n+1 / m}^{0}$.

Proof. From the definition of class $K_{3}(\mathcal{D})$, if $T \in K_{3}(\mathcal{D})$ and $\mathcal{D}^{*}$ is any domain within $\mathcal{D}$, then $T \in K_{3}\left(\mathscr{D}^{*}\right)$. See Radó and Reichelderfer [1].

3.4. LEMma. If $T$ satisfies $H_{0}$, the transformation

$$
{ }_{n}^{n} T: \quad x={ }_{n}^{n} x(u, v), \quad y=y(u, v), \quad n>2,(u, v) \in{ }_{n} S,
$$

belongs to class $K_{3}$ on ${ }_{n} S^{0}$.

Proof. Fix a value of $n>2$. For each positive integer $m$, satisfying $1 / n+1 / m<1 / 2$, define the transformation ${ }_{n}^{n} T_{m}^{m}$ on $S_{1 / n+1 / m}$. We shall verify that the transformations ${ }_{n}^{n} T$ and ${ }_{n}^{n} T_{m}^{m}$ satisfy the conditions of the Closure Theorem (cf. 1.7).

(i) Clearly $S_{1 / n+1 / m}^{0} \subset_{n} S^{0}$ and, for every closed set $F C_{n} S^{0}$, there exists an $m_{0}=m_{0}(F)$ such that $F \subset S_{1 / n+1 / m}^{0}$ for all $m>m_{0}$.

(ii) By 2.8, $\partial_{n}^{n} x(u, v) / \partial u$ and $\partial_{n}^{n} x(u, v) / \partial v$ are continuous on ${ }_{n} S$ and, therefore, bounded. The summability of ${ }^{n} J=J\left(u, v ;{ }_{n}^{n} T\right)$ on ${ }_{n} S^{0}$ follows then from the summability of $y_{u}(u, v)$ and $y_{v}(u, v)$ on $S_{0}$ (cf. 2.18).

(iii) By 3.3.2, ${ }_{n}^{n} T_{m}^{m} \in K_{3}\left(S_{1 / n+1 / m}^{0}\right)$ for $1 / n+1 / m<1 / 2$.

(iv) For every closed set $F C_{n} S^{0}$ there exists a closed oriented rectangle $R$ such that $F \subset R \subset_{n} S^{0}$. By $2.7,{ }_{m}^{m} y(u, v) \rightarrow_{m}$ uniformly to $y(u, v)$ on $R$. To show that ${ }^{n} J^{m}$ converges strongly to ${ }^{n} J$ on $F$, we shall verify that ${ }^{n} J^{m} \rightarrow_{m}{ }^{n} J$ a.e. on $R$ and that $\left\{{ }^{n} J^{m}\right\}$ has property $(U)$ on $R$ for fixed $n$; hence, by 2.3.1, $\left.\iint_{R}\right|^{n} J^{m}-{ }^{n} J \mid d u d v \rightarrow_{m} 0$.

It follows directly from 2.19 .1 that ${ }^{n} J^{m} \rightarrow_{m}{ }^{n} J$ a.e. on $R$. To show property (U) for $\left\{{ }^{n} J^{m}\right\}$ on $R$, it suffices to consider the product

$$
\frac{\partial_{n}^{n} x(u, v)}{\partial u} \frac{\partial_{m}^{m} y(u, v)}{\partial v}=\frac{\partial_{n}^{n} x(u, v)}{\partial u}{ }_{m}^{m}\left(\frac{\partial y(u, v)}{\partial v}\right)
$$

since the other product of ${ }^{n} J^{m}$ may be handled in the same way. By 2.8 , the first factor is bounded for fixed $n$ and, by $2.10,\left\{{ }_{m}^{m}(\partial y(u, v) / \partial v)\right\}$ has property $(U)$ on $R$; hence, from 2.2.2, $\left\{\partial_{n}^{n} x(u, v) / \partial u \cdot \partial_{m}^{m} y(u, v) / \partial v\right\}$ has property $(U)$ on $R$ for fixed $n$.

The conditions of the Closure Theorem being satisfied, we conclude that ${ }_{n}^{n} T \in K_{3}\left({ }_{n} S^{0}\right)$.

Exchanging the rôles of $x(u, v)$ and $y(u, v)$, we have the lemma:

3.4.1. Lемма. If $T$ satisfies $H_{0}$, the transformation

$$
T_{n}^{n}: \quad x=x(u, v), \quad y={ }_{n}^{n} y(u, v), \quad n>2,(u, v) \in{ }_{n} S,
$$

belongs to class $K_{3}$ on ${ }_{n} S^{0}$. 
3.5. Our investigation of the transformations considered by Young will proceed according to the following scheme: First we approximate to the given transformation $T$ by a suitable one of the transformations ${ }_{n}^{n} T_{n}^{n}{ }_{n}^{n} T, T_{n}^{n}$. Then, as the reader will easily verify, all of the conditions of the Closure Theorem are satisfied except strong convergence of the Jacobians. Since the Jacobians of the approximating transformations converge a.e. to $J(u, v ; T)$ (cf. 2.19.1), a necessary and sufficient condition for strong convergence is that the Jacobians of the approximating transformations have property (U) (cf. 2.2, 2.3.1). We shall now investigate property $(U)$ for the Jacobians of the approximating transformations.

3.6. Theorem 1 (see Young [2, pp. 80-85], cf. 1.9). If T satisfies $H_{0}$ and one factor in each product $x_{u}(u, v) \cdot y_{v}(u, v), x_{v}(u, v) \cdot y_{u}(u, v)$ is bounded on $S_{0}$, then $T \in K_{3}\left(S_{0}^{0}\right)$ and formula (1) holds whenever the integral on the left exists.

Proof. Consider any fixed $R \subset S_{0}^{0}$. Choose $n$ large enough that $R C_{n} S$. Approximate to $T$ by ${ }_{n}^{n} T_{n}^{n}$. To show property $(U)$ for $\left\{{ }^{n} J^{n}\right\}$ on $R$, it is sufficient to consider the product

$$
\frac{\partial_{n}^{n} x(u, v)}{\partial u} \frac{\partial_{n}^{n} y(u, v)}{\partial v}={ }_{n}^{n}\left(x_{u}(u, v)\right) \cdot{ }_{n}^{n}\left(y_{v}(u, v)\right)
$$

since the other product in ${ }^{n} J^{n}$ may be handled in the same way. By assumption, one factor of $x_{u}(u, v) \cdot y_{v}(u, v)$, say $y_{v}(u, v)$, is bounded on $S_{0}$; hence, ${ }_{n}^{n}\left(y_{v}(u, v)\right)$ is uniformly bounded on $R$. By $2.10,\left\{{ }_{n}^{n}\left(x_{u}(u, v)\right)\right\}$ has property (U) on $R$. Therefore, from 2.2.2, $\left\{\partial_{n}^{n} x(u, v) / \partial u \cdot \partial_{n}^{n} y(u, v) / \partial v\right\}$ has property (U) on $R$. By the Closure Theorem, $T \in K_{3}\left(S_{0}^{0}\right)$ and, by the result of Radó and Reichelderfer mentioned in 1.2, the theorem follows.

3.7. Theorem 2 (see Young [2, pp. 80-85], cf. 1.9). If T satisfies $H_{0}$ and in each product $\dot{x}_{u}(u, v) \cdot y_{v}(u, v), x_{v}(u, v) \cdot y_{u}(u, v)$, the factors belong to associated Lebesgue classes on $S_{0}$, then $T \in K_{3}\left(S_{0}^{0}\right)$ and formula (1) holds providing the integral on the left exists.

Proof. Consider any fixed $R \subset S_{0}^{0}$. Choose $n$ large enough that $R \subset_{n} S$. Approximate to $T$ by ${ }_{n}^{n} T_{n}^{n}$. On $R$

$$
\left|{ }_{n}^{n} J_{n}^{n}\right| \leqq\left|{ }_{n}^{n}\left(x_{u}(u, v)\right) \cdot{ }_{n}^{n}\left(y_{v}(u, v)\right)\right|+\left|{ }_{n}^{n}\left(x_{v}(u, v)\right) \cdot{ }_{n}^{n}\left(y_{u}(u, v)\right)\right| \text {. }
$$

It now follows from 2.11 and 2.2.1 that $\left\{{ }^{n} J^{n}\right\}$ has property $(U)$ on $R$. By the Closure Theorem, $T \in K_{3}\left(S_{0}^{0}\right)$; hence, the theorem follows.

3.8. Theorem 3 (see Young [3, p. 163], cf. 1.9). If T satisfies $H_{0} ;\left|x_{u}(u, v)\right|$, $\left|y_{u}(u, v)\right| \leqq \lambda(u)$ and $\left|x_{v}(u, v)\right|,\left|y_{v}(u, v)\right| \leqq \mu(v)$, where $\lambda(u)$ and $\mu(v)$ are summable on $(0,1)$, then $T \in K_{3}\left(S_{0}^{0}\right)$ and formula (1) holds as soon as the integral on the left exists. 
Proof. Consider any fixed $R \subset S_{0}^{0}$. Choose $n$ large enough that $R C_{n} S$. Approximate to $T$ by ${ }_{n}^{n} T$. To show property $(U)$ for $\left\{{ }^{n} J\right\}$ on $R$, it suffices to consider the product $\partial_{n}^{n} x(u, v) / \partial u \cdot \partial y(u, v) / \partial v$ since the other product of ${ }^{n} J$ may be handled in the same way. Let $e$ be any measurable set comprised in $R$. Then

$$
\begin{aligned}
& \iint_{e}\left|\frac{\partial_{n}^{n} x(u, v)}{\partial u}\right| \cdot\left|\frac{\partial y(u, v)}{\partial v}\right| d u d v \\
& \leqq \frac{n^{2}}{4} \int_{-1 / n}^{1 / n} \int_{-1 / n}^{1 / n}\left\{\iint_{e}\left|x_{u}(u+\alpha, v+\beta)\right| \cdot\left|y_{v}(u, v)\right| d u d v\right\} d \alpha d \beta \\
& \leqq \frac{n^{2}}{4} \int_{-1 / n}^{1 / n} \int_{-1 / n}^{1 / n}\left\{\iint_{e} \lambda(u+\alpha) \mu(v) d u d v\right\} d \alpha d \beta \\
& \leqq \frac{n^{2}}{4} \int_{-1 / n}^{1 / n} \int_{-1 / n}^{1 / n}\left\{\iint_{e_{\alpha}} \lambda(u) \mu(v) d u d v\right\} d \alpha d \beta \\
& \leqq \frac{n^{2}}{4} \int_{-1 / n}^{1 / n} \int_{-1 / n}^{1 / n} \Omega\left(\lambda(u) \mu(v), S_{0},|e|\right) d \alpha d \beta \\
& \leqq \Omega\left(\lambda(u) \mu(v), S_{0},|e|\right) \text {. }
\end{aligned}
$$

$\Omega$ has been defined in 2.10 and

$$
e_{\alpha}=\underset{(u, v)}{E}[(u-\alpha, v) \in e]
$$

By the Theorem of Tonelli, $\lambda(u) \mu(v)$ is summable on $S_{0}$; hence, $\Omega\left(\lambda(u) \mu(v), S_{0}\right.$, $|e|) \rightarrow_{|\epsilon| \rightarrow 0} 0$. Thus, by the last inequality and 2.2.1, $\left\{\partial_{n}^{n} x(u, v) / \partial u \cdot \partial y(u, v) / \partial v\right\}$ has property $(U)$ on $R$. Finally, by the Closure Theorem, $T \in K_{3}\left(S_{0}^{0}\right)$ and the theorem follows.

3.9. THEOREM 4 (cf. 3.1). If $T$ satisfies $H_{0} ;\left|x_{u}(u, v)\right| \leqq \lambda(u),\left|x_{v}(u, v)\right|$ $\leqq \mu(v)$, where $\lambda(u)$ and $\mu(v)$ are summable on $(0,1)$; and the total variations $V(v ; y)$ and $V(u ; y)$ are bounded on $(0,1)$, then $T \in K_{3}\left(S_{0}^{0}\right)$ and formula (1) holds whenever the integral on the left exists.

Proof. Consider any fixed $R \subset S_{0}^{0}$. Choose $n$ large enough that $R C_{n} S$. Approximate to $T$ by $T_{n}$. On $R,\left|J^{n}\right| \leqq \lambda(u) \cdot{ }_{n}^{n}\left|y_{v}(u, v)\right|+\mu(v) \cdot{ }_{n}^{n}\left|y_{u}(u, v)\right|$. This inequality, along with $2.14,2.3$, and 2.2 .1 , implies property $(U)$ for $\left\{J^{n}\right\}$ on $R$. By the Closure Theorem, $T \in K_{3}\left(S_{0}^{0}\right)$, so the theorem follows.

3.10. Young really does not discuss a situation corresponding to Theorem 4. Instead, he claims (see Young [2, p. 88]) to have established formula (3) of 1.4 under the conditions: $x(u, v)$ and $y(u, v)$ are absolutely continuous on every horizontal and on every vertical in $S_{0} ; y_{u}(u, v), y_{v}(u, v)$ are summable on $S_{0} ;\left|x_{u}(u, v)\right| \leqq \lambda(u)$ and $\left|x_{v}(u, v)\right| \leqq \mu(v)$, where $\lambda(u), \mu(v)$ are summable on $(0,1)$ and $\lambda(u) y_{v}(u, v), \mu(v) y_{u}(u, v)$ are summable on $S_{0}$. In attempting to prove this assertion, Young erroneously assumes that $y(u, v)$ can be written 
as follows: $y(u, v)=y_{1}(u, v)-y_{2}(u, v)$, where $y_{1}(u, v)$ and $y_{2}(u, v)$ possess nonnegative first partial derivatives in addition to all of the properties of $y(u, v)$ (see Young [2, p. 91]). Even if $y(u, v)$ could be split in this manner, $V(v ; y)$ and $V(u ; y)$ would be bounded (as we assumed in Theorem 4) since

$$
\begin{aligned}
V(v ; y) & \leqq V\left(v ; y_{1}\right)+V\left(v ; y_{2}\right)=\int_{0}^{1} \frac{\partial y_{1}(u, v)}{\partial u} d u+\int_{0}^{1} \frac{\partial y_{2}(u, v)}{\partial u} d u \\
& \leqq y_{1}(1, v)-y_{1}(0, v)+y_{2}(1, v)-y_{2}(0, v)
\end{aligned}
$$

and, similarly, $V(u ; y) \leqq y_{1}(u, 1)-y_{1}(u, 0)+y_{2}(u, 1)-y_{2}(u, 0)$.

3.11. For the purpose of the next theorem, we require the following lemma:

LEMMA. If $f(u, v) \geqq 0$ and $g(u, v) \geqq 0$ are summable on $S_{0}$ and if $0<\delta<1 / 2$ is fixed, then

$$
\phi(\alpha, \beta)=\iint_{S_{\delta}} f(u+\alpha, v+\beta) g(u, v) d u d v
$$

exists a.e. and is summable in $r:-\delta \leqq \alpha \leqq \delta,-\delta \leqq \beta \leqq \delta$.

Proof. See Young [2, p. 91].

3.12. Theorem 5 (see Young [2, pp. 92-93]; cf. 1.9, 3.1). For T satisfying $H_{0}$ and $0<\delta<1 / 2$ fixed, define

$T(\alpha, \beta ; 0,0): \quad x=x(u+\alpha, v+\beta), \quad y=y(u, v), \quad(\alpha, \beta) \in r,(u, v) \in S_{8}$.

Then for a.e. $(\alpha, \beta)$ in $r, T(\alpha, \beta ; 0,0) \in K_{3}\left(S_{\delta}^{0}\right)$ and the transformation formula

$$
\begin{aligned}
\iint_{S_{\delta}} F(x(u+\alpha, v+\beta), y & (u, v)) J(u, v ; T(\alpha, \beta ; 0,0)) d u d v \\
& =\int_{-\infty}^{+\infty} \int_{-\infty}^{+\infty} F(x, y) \cdot \mu\left(x, y ; S_{\delta}, T(\alpha, \beta ; 0,0)\right) d x d y
\end{aligned}
$$

holds if the integral on the left exists.

Proof. Consider any fixed $R \subset S_{\delta}^{0}$. Choose $n_{0}$ large enough that $R \subset S_{\delta+1 / n}$ for $n>n_{0}$. Then for $(\alpha, \beta) \in r$ and $n>n_{0}$, define

$$
{ }_{n}^{n} T(\alpha, \beta ; 0,0): \quad x={ }_{n}^{n} x(u+\alpha, v+\beta), \quad y=y(u, v), \quad(u, v) \in S_{\delta+1 / n} .
$$

We wish to show that $\left\{J\left(u, v ;{ }_{n}^{n} T(\alpha, \beta ; 0,0)\right\}\right.$ has property $(U)$ on $R$ for a.e. $(\alpha, \beta)$ in $r$. To this end, we need only consider the product $\partial_{n}^{n} x(u+\alpha, v+\beta) / \partial u$ $\cdot \partial y(u, v) / \partial v$ since the other product of the Jacobian may be handled in the same way.

For $(\alpha, \beta) \in r$, define 


$$
G(u, v, \alpha, \beta)=\left|x_{u}(u+\alpha, v+\beta)\right| \cdot\left|y_{v}(u, v)\right|, \quad(u, v) \in S_{\delta},
$$

and

$G_{n}(u, v, \alpha, \beta)={ }_{n}^{n}\left|x_{u}(u+\alpha, v+\beta)\right| \cdot\left|y_{v}(u, v)\right|, \quad n>n_{0},(u, v) \in S_{\delta+1 / n}$.

By 3.11,

$$
H(\alpha, \beta)=\iint_{R} G(u, v, \alpha, \beta) d u d v
$$

exists a.e. and is summable on $r$. Let $E$ be the exceptional set of points $(\alpha, \beta)$ in $r$. From 2.9,

$$
{ }_{n}^{n} H(\alpha, \beta)=\iint_{R} G_{n}(u, v, \alpha, \beta) d u d v \underset{n}{\rightarrow} \iint_{R} G(u, v, \alpha, \beta) d u d v=F(\alpha, \beta)
$$

for a.e. $(\alpha, \beta) \in r$. Let $E_{2}$ be the exceptional set of points $(\alpha, \beta)$ in $r$. Consider a fixed point $\left(\alpha_{0}, \beta_{0}\right)$ in $r$ and not belonging to $E_{1}+E_{2}$. By 2.9, $G_{n}\left(u, v, \alpha_{0}, \beta_{0}\right) \rightarrow_{n} G\left(u, v, \alpha_{0}, \beta_{0}\right)$ a.e. on $R$; hence, it follows from (1) and 2.3 that $\left\{G_{n}\left(u, v, \alpha_{0}, \beta_{0}\right)\right\}$ has property $(U)$ on $R$. For $(u, v) \in R$ and $n>n_{0}$,

$$
G_{n}\left(u, v, \alpha_{0}, \beta_{0}\right) \geqq\left|\frac{\partial_{n}^{n} x\left(u+\alpha_{0}, v+\beta_{0}\right)}{\partial u}\right| \cdot\left|\frac{\partial y(u, v)}{\partial v}\right| ;
$$

therefore, $\left\{\partial_{n}^{n} x\left(u+\alpha_{0}, v+\beta_{0}\right) / \partial u \cdot \partial y(u, v) / \partial v\right\}$ has property $(U)$ on $R$. Finally, by the Closure Theorem, $T(\alpha, \beta ; 0,0)$ belongs to class $K_{3}$ on $S_{\delta}^{0}$ for a.e. $(\alpha, \beta)$ in $r$.

To complete the theorem, we must show that for a.e. $(\alpha, \beta)$ in $r$ the image of the boundary of $S_{\delta}$ under $T(\alpha, \beta ; 0,0)$ is a set of planar measure zero. Since $x(u, v)$ is A.C.T. on $S_{0}$, for a.e. $(\alpha, \beta)$ in $r$ the function $x(u+\alpha, v+\beta)$ is absolutely continuous on the boundary of $S_{\delta}$; hence, for such $(\alpha, \beta)$, the continuous image of the boundary of $S_{\delta}$ under $T(\alpha, \beta ; 0,0)$ is a semi-rectifiable curve and its locus is a set of planar measure zero.

(For the proof of Theorem 5 we need not assume that the image of the boundary of $S_{0}$ under $T$ is a set of planar measure zero.)

3.13. To prove the final theorem, we approximate to the given transformation $T$ by the transformation

$$
{ }_{n} T_{n}: \quad x={ }_{n} x(u, v), \quad y={ }_{n} y(u, v), \quad n>2,(u, v) \in R_{1 / n 0} .
$$

Thus, to utilize the Closure Theorem, we must establish the lemma:

LEMмA. If $T$ satisfies $H_{0}$, the transformation ${ }_{n} T_{n}$ belongs to class $K_{3}$ on $R_{1 / n}^{0}$.

Proof. By 2.22, ${ }_{n} x(u, v)$ and ${ }_{n} y(u, v)$ are A.C.T. on $R_{1 / n}$. From 2.15, $\partial_{n} x(u, v) / \partial u$ and $\partial_{n} y(u, v) / \partial u$ are continuous and, therefore, bounded on $R_{1 / n}$ 0. The result now follows from reasoning similar to that employed in 3.6. 
3.14. Theorem 6 (see Young [1, pp. 365-366; 2, p. 87]; cf. 1.9, 3.1). If $T$ satisfies $H_{0}$ and $\left|x_{v}(u, v)\right|,\left|y_{v}(y, v)\right| \leqq \mu(v)$, where $\mu(v) x_{u}(u, v)$ and $\mu(v) y_{u}(u, v)$ are summable on $S_{0}$, then $T \in K_{3}\left(S_{0}^{0}\right)$ and formula (1) holds providing the integral on the left exists.

Proof. Consider any fixed $R \subset S_{0}^{0}$. Choose $n$ large enough that $R \subset R_{1 / n} 0$. Approximate to $T$ by ${ }_{n} T_{n}$. To show property $(U)$ for $\left\{J\left(u, v ;{ }_{n} T_{n}\right)\right\}$ on $R$, it suffices to consider the product $\partial_{n} x(u, v) / \partial u \cdot \partial_{n} y(u, v) / \partial v$ since the other product of the Jacobian may be handled in the same way. Let $e$ be any measurable set comprised in $R$. Then

$$
\begin{aligned}
& \iint_{e}\left|\frac{\partial_{n} x(u, v)}{\partial u}\right| \cdot\left|\frac{\partial_{n} y(u, v)}{\partial v}\right| d u d v \\
& \leqq \frac{n^{2}}{4} \int_{-1 / n}^{1 / n} \int_{-1 / n}^{1 / n}\left\{\iint_{e}\left|x_{u}(u+\alpha, v)\right| \cdot\left|y_{v}(u+\beta, v)\right| d u d v\right\} d \alpha d \beta \\
& \leqq \frac{n^{2}}{4} \int_{-1 / n}^{1 / n} \int_{-1 / n}^{1 / n}\left\{\iint_{e} \mu(v)\left|x_{u}(u+\alpha, v)\right| d u d v\right\} d \alpha d \beta \\
& \leqq \frac{n^{2}}{4} \int_{-1 / n}^{1 / n} \int_{-1 / n}^{1 / n}\left\{\iint_{e_{\alpha}} \mu(v)\left|x_{u}(u, v)\right| d u d v\right\} d \alpha d \beta \\
& \leqq \frac{n^{2}}{4} \int_{-1 / n}^{1 / n} \int_{-1 / n}^{1 / n} \Omega\left(\mu(v)\left|x_{u}(u, v)\right|, S_{0},|e|\right) d \alpha d \beta \\
& \leqq \Omega\left(\mu(v)\left|x_{u}(u, v)\right|, S_{0},|e|\right),
\end{aligned}
$$

where $\Omega\left(\mu(v)\left|x_{u}(u, v)\right|, S_{0},|e|\right) \rightarrow_{|e| \rightarrow 0} 0$. Thus, by the last inequality and 2.2.1, $\left\{\partial_{n} x(u, v) / \partial u \cdot \partial_{n} y(u, v) / \partial v\right\}$ has property $(U)$ on $R$.

From 2.20.1 and 2.21.1, $J\left(u, v ;{ }_{n} T_{n}\right) \rightarrow_{n} J(u, v ; T)$ a.e. on $R$; hence, as the reader can now easily verify, all of the conditions of the Closure Theorem are fulfilled. Therefore, $T \in K_{3}\left(S_{0}^{0}\right)$ and the theorem follows.

3.14.1. Corollary (see Young [3, pp. 189-190], cf. 1.9). If T satisfies $H_{0}$; $\left|x_{v}(u, v)\right|,\left|y_{v}(u, v)\right| \leqq \mu(v)$, where $\mu(v)$ is summable on $(0,1)$; and the total variations $V(v ; x)$ and $V(v ; y)$ are bounded on $(0,1)$, then $T \in K_{3}\left(S_{0}^{0}\right)$ and formula (1) holds as soon as the integral on the left exists.

Proof. Because of the Theorem of Tonelli, the relation

$$
\int_{0}^{1}\left\{\int_{0}^{1} \mu(v)\left|x_{u}(u, v)\right| d u\right\} d v=\int_{0}^{1} \mu(v) V(v ; x) d v
$$

implies the summability of $\mu(v) x_{u}(u, v)$ on $S_{0}$, Likewise, $\mu(v) y_{u}(u, v)$ is summable on $S_{0}$.

3.15. The preceding six theorems account for all of Young's results on the 
transformation of double integrals. Theorem 5 seems to offer a method of approach to the conjectured proposition (cf. 1.10):

If $T$ satisfies $H_{0}$ and $J(u, v ; T)$ is summable on $S_{0}$, then $T \in K_{3}\left(S_{0}^{0}\right)$ and formula (1) holds if the integral on the left exists.

Along this line, we have considered the question of deducing Theorems 1, $2,3,4$, and 6 from Theorem 5 . The first four theorems follow readily from the fifth, but we have not been able to deduce Theorem 6 from Theorem 5 .

\section{BiBLIOGRAPHY}

H. E. Bray

1. Proof of a formula for an area, Bull. Amer. Math. Soc. vol. 29 (1923) pp. 264-270.

G. FUBINI

1. Sulla derivata seconda mista di un integrale doppio, Rend. Cir. Mat. Palermo vol. 40 (1915) pp. 297- 98

R. G. Helsel ANd P. M. Young

1. Characterization theorems for integral means, to appear soon in Duke Math. J.

E. J. McShane

1. Integrals over surfaces in parametric form, Ann. of Math. (2) vol. 34 (1933) pp. 815-838.

C. B. MORREY

1. A class of representations of manifolds. I, Amer. J. Math. vol. 55 (1933) pp. 683-707.

H. RADEMACHER

1. Über partielle und totale Differenzierbarkeit von Funktionen mehrerer Variabeln. I, Math. Ann. vol. 79 (1919) pp. 340-359.

T. RADó

1. On continuous transformations in the plane, Fund. Math. vol. 27 (1936) pp. 201-211.

2. A remark on the area of surfaces, Amer. J. Math. vol. 58 (1936) pp. 598-606.

3. On absolutely continuous transformations in the plane, Duke Math. J. vol. 4 (1938) pp. 189-221.

T. RADó AND P. REICHELDERFER

1. A theory of absolutely continuous transformations in the plane, Trans. Amer. Math. Soc. vol. 49 (1941) pp. 258-307.

J. SCHAUdER

1. Über stetige Abbildungen, Fund. Math. vol. 12 (1928) pp. 47-74.

C. DE la Vallée Poussin

1. Sur l'intégrale de Lebesgue, Trans. Amer. Math. Soc. vol. 16 (1915) pp. 435-501.

W. H. Young

1. On a formula for an area, Proc. London Math. Soc. vol. 18 (1920) pp. 339-374.

2. On a new set of conditions for a formula for an area, Proc. London Math. Soc. vol. 21 (1923) pp. 75-94.

3. Integration over the area of a curve and transformation of the variables in a multiple integral, Proc. London Math. Soc. vol. 21 (1923) pp. 161-190.

The Ohio State University, Columbus, Ohio. 